\title{
A Note on Locally Inverse Semigroup Algebras
}

\author{
Xiaojiang Guo \\ Department of Mathematics, Jiangxi Normal University, Nanchang, Jiangxi 330022, China
}

Correspondence should be addressed to Xiaojiang Guo, xjguo@jxnu.edu.cn

Received 9 December 2007; Accepted 29 January 2008

Recommended by Francois Goichot

Let $R$ be a commutative ring and $S$ a finite locally inverse semigroup. It is proved that the semigroup algebra $R[S]$ is isomorphic to the direct product of Munn algebras $\mathcal{M}\left(R\left[G_{J}\right], m_{J}, n_{J} ; P_{J}\right)$ with $J \in S / \mathcal{2}$, where $m_{J}$ is the number of $\mathcal{R}$-classes in $J, n_{J}$ the number of $\mathcal{L}$-classes in $J$, and $G_{J}$ a maximum subgroup of $J$. As applications, we obtain the sufficient and necessary conditions for the semigroup algebra of a finite locally inverse semigroup to be semisimple.

Copyright (C) 2008 Xiaojiang Guo. This is an open access article distributed under the Creative Commons Attribution License, which permits unrestricted use, distribution, and reproduction in any medium, provided the original work is properly cited.

\section{Main results}

A regular semigroup $S$ is called a locally inverse semigroup if for all idempotent $e \in S$, the local submonoid $e S e$ is an inverse semigroup under the multiplication of $S$. Inverse semigroups are locally inverse semigroups. Inverse semigroup algebras are a class of semigroup algebras which is widely investigated. One of fundamentally important results is that a finite inverse semigroup algebra is the direct product of full matrix algebras over group algebras of the maximum subgroups of this finite inverse semigroup. Consider that all local submonoids of a locally inverse semigroup are inverse semigroups, it is a very natural problem whether a finite locally inverse semigroup algebra has a similar representation to inverse semigroup algebras. This is the main topic of this note.

Let $A$ be an $R$-algebra. Let $m$ and $n$ be positive integers, and let $P$ be a fixed $n \times m$ matrix over $\mathcal{A}$. Let $\mathcal{M}:=\mathcal{M}(\mathcal{A} ; m, n ; P)$ be the vector space of all $m \times n$ matrices over $\mathcal{A}$. Define a product $\circ$ in $\mathcal{M}$ by

$$
A \circ B=A P B \quad(A, B \in \mathcal{M}),
$$

where $A P B$ is the usual matrix product of $A, P$, and $B$. Then $\mathcal{M}$ is an algebra over $R$. Following [1], we call $\mathcal{M}$ the Munn $m \times n$ matrix algebra over $\&$ with sandwich matrix $P$. 
By a semisimple semigroup, we mean a semigroup each of whose principal factor is either a completely 0 -simple semigroup or a completely simple semigroup. It is well known that a finite regular semigroup is semisimple. The Rees theorem tells us that any completely 0 -simple semigroup (completely simple semigroup) is isomorphic to some Rees matrix semigroup $\mathcal{M}^{0}(G, I, \Lambda ; P)(\mathcal{M}(G, I, \Lambda ; P))$, and vice versa (for Rees matrix semigroups, refer to [1]). In what follows, by the phrase "Let $S=\bigcup_{J \in S / 2} \mathcal{M}^{0}\left(G_{J} ; I_{J}, \Lambda_{J} ; P_{J}\right)$ be a finite regular semigroup," we mean that $S$ is a finite regular semigroup in which the principal factor of $S$ determined by the 2 -class $J$ is isomorphic to the Rees matrix semigroup $\mathcal{M}^{0}\left(G_{J} ; I_{J}, \Lambda_{J} ; P_{J}\right)$ or $\mathcal{M}\left(G_{J} ; I_{J}, \Lambda_{J} ; P_{J}\right)$ for any $J \in S / 2$.

The following is the main result of this paper.

Theorem 1.1. Let $S=\bigcup_{J \in S / 2} \mathcal{M}^{0}\left(G_{J}, I_{J}, \Lambda_{J} ; P_{J}\right)$ be a finite locally inverse semigroup. Then the semigroup algebra $R[S]$ is isomorphic to the direct product of $\mathcal{M}\left(R\left[G_{J}\right] ;\left|I_{J}\right|,\left|\Lambda_{J}\right| ; P_{J}\right)$ with $J \in S / 2$.

Based on Theorem 1.1 and [1, Lemma 5.17, page 162, and Lemma 5.18, page 163], the following corollary is straightforward.

Corollary 1.2. Let $S=\bigcup_{J \in S / 2} \mathcal{M}^{0}\left(G_{J}, I_{J}, \Lambda_{J} ; P_{J}\right)$ be a finite locally inverse semigroup. Then the semigroup algebra $R[S]$ has an identity if and only if $\left|I_{J}\right|=\left|\Lambda_{J}\right|$ and $P_{J}$ is invertible in the full matrix algebra $M_{\left|I_{J}\right|}\left(R\left[G_{J}\right]\right)$ for all $J \in S / 2$.

Reference [1, Lemma 5.18, page 163] told us that $\mathcal{M}\left(R\left[G_{J}\right], m_{J}, n_{J} ; P_{J}\right)$ is isomorphic to the full matrix algebra $M_{n_{J}}\left(R\left[G_{J}\right]\right)$ if $\mathcal{M}\left(R\left[G_{J}\right], m_{J}, n_{J} ; P_{J}\right)$ has an identity. Now, we have the following.

Corollary 1.3. Let $S=\bigcup_{J \in S / 2} \mathcal{M}^{0}\left(G_{J}, I_{J}, \Lambda_{J} ; P_{J}\right)$ be a finite locally inverse semigroup. If $R[S]$ has an identity, then $R[S]$ is isomorphic to the direct product of the full matrix algebras $M_{\left|I_{J}\right|}\left(R\left[G_{J}\right]\right)$ with $J \in S / 2$.

The following corollary is a consequence of Corollary 1.3.

Corollary 1.4. Let $S=\bigcup_{J \in S / 2} \mathcal{M}^{0}\left(G_{J}, I_{J}, \Lambda_{J} ; P_{J}\right)$ be a finite locally inverse semigroup. Then the semigroup algebra $R[S]$ is semisimple if and only if for all $J \in S / 2$,

(1) $\left|I_{J}\right|=\left|\Lambda_{J}\right|$;

(2) $P_{J}$ is invertible in the full matrix algebra $M_{\left|I_{J}\right|}\left(R\left[G_{J}\right]\right)$;

(3) $R\left[G_{J}\right]$ is semisimple.

\section{Proof of Theorem 1.1}

For our purpose, we have the Möbius inversion theorem [2].

Lemma 2.1. Let $(P, \leq)$ be a locally finite partially ordered set (i.e., intervals are finite) in which each principal ideal has a maximum and $G$ be an Abelian group. Suppose that $f: P \rightarrow G$ is a function and define $g: P \rightarrow G$ by $g(x)=\sum_{y \leq x} f(y)$. Then $f(x)=\sum_{y \leq x} g(y) \mu(x, y)$, where $\mu$ is a Möbius function.

Now assume that $S$ is a regular semigroup and $a, b \in S$. Define

$$
a \leq b \Longleftrightarrow \text { there exist } e, f \in E(S) \text { such that } a=e b=b f \text {. }
$$


Then $\leq$ is a partial order on $S$. Following [3], we call $\leq$ the natural partial order on $S$. Equivalently, $a \leq b$ if and only if for every (for some) $f \in E\left(R_{b}\right)\left(f \in E\left(L_{b}\right)\right)$, there exists $e \in$ $E\left(R_{a}\right)\left(e \in E\left(L_{a}\right)\right)$ such that $e \leq f$ and $a=e b(a=b e)$. Moreover, Nambooripad [3, 4] proved that $S$ is a locally inverse semigroup if and only if the natural partial order $\leq$ is compatible with respect to the multiplication of $S$.

Lemma 2.2. Let $S$ be a locally inverse semigroup and $a, b \in S$. Then for any $u \leq a b$, there exist $x \leq a$ and $y \leq b$ such that $u=x y, x \in R_{u}$ and $y \in L_{u}$.

Proof. For any $e \in E\left(R_{a}\right)$, we have $e a=a$ and $e a b=a b$. Let $z$ be an inverse of $a b$. Clearly, $a b z \in E\left(R_{a b}\right)$. Note that $e a b z=a b z$. It is easy to check that abze $\in E(S)$, abze $\leq e$, and $a b z R a b z e$. Hence $a b z e R a b$ and there exists $g \in E(S)$ such that $u=g a b$ and $g \leq a b z e(\leq e)$. Thus $g a \leq a$. On the other hand, since $R$ is a left congruence and since $a b z e R a b$, we have $u=g a b R g a b z e=g$; while since $a \mathcal{R} e$, we have $g a R g e=g$. These imply that $u R g a$. Dually, we have $h \in E(S)$ such that $u=a b h, b h \leq b$ and $u \perp b h$. Since $u=g a b=a b h=u h=(g a)(b h)$, we know that $g a$ and $b h$ are the required elements $x$ and $y$.

Define a multiplication $\otimes$ on $S^{0}=S \cup\{0\}$ by

$$
x \otimes y= \begin{cases}x y & \text { if } x \neq 0, y \neq 0, \text { and } y, x y \in J_{x} \\ 0 & \text { otherwise }\end{cases}
$$

where $x y$ is the product of $x$ and $y$ in $S$. By the arguments of $[4$, page 9$],\left(S^{0}, \otimes\right)$ is a semigroup. We denote by $S^{\otimes}$ the semigroup $\left(S^{0}, \otimes\right)$. For any $J \in S / 2$, we denote $J^{0}=J \cup\{0\}$. It is easy to check that $\left(J^{0}, \otimes\right)$ is a subsemigroup of $S^{\otimes}$, which is isomorphic to the principal factor of $S$ determined by $J$. We will denote the semigroup $\left(J^{0}, \otimes\right)$ by $J^{\otimes}$. By the definition of $\otimes$, it is easy to see that in the semigroup $S^{\otimes}$,

(i) $J_{x}^{\otimes} \otimes J_{x}^{\otimes} \subseteq J_{x}^{\otimes}$ for all $x \in S$;

(ii) $J_{x}^{\otimes} \otimes J_{y}^{\otimes}=0$ for all $x, y \in S$ such that $x \notin J_{y}$.

Thus $R_{0}\left[S^{\otimes}\right]$ is the direct sum of the contracted semigroup algebras $R_{0}\left[J^{\otimes}\right]$ with $J \in S / 2$. Note that $J^{\otimes}$ is isomorphic to some principal factor of $S$. We observe that $J^{\otimes}$ is a completely 0 simple semigroup since $S$ is a semisimple semigroup, and thus $J^{\otimes}$ is isomorphic to some Rees matrix semigroup $\mathcal{M}^{0}\left(G_{J}, I_{J}, \Lambda_{J} ; P_{J}\right)$. By a result of $[1], R_{0}\left[\mathcal{M}^{0}\left(G_{J}, I_{J}, \Lambda_{J} ; P_{J}\right)\right]$ is isomorphic to $\mathcal{M}\left(R\left[G_{J}\right],\left|I_{J}\right|,\left|\Lambda_{J}\right| ; P_{J}\right)$. Consequently, to verify Theorem 1.1 , we need only to prove that $R[S]$ is isomorphic to $R_{0}\left[S^{\otimes}\right]$.

For the convenience of description, we introduce the semigroup $\bar{S}$. Put $\bar{S}=\{\bar{x} \mid x \in$ $S\} \cup\{0\}$. Define a multiplication on $\bar{S}$ as follows:

$$
\bar{x} * \bar{y}=\overline{x \otimes y},
$$

where we will identify $\overline{0}$ with 0 . It is easy to see that $\bar{S}$ is isomorphic to $S^{\otimes}$. Hence the contracted semigroup algebra $R_{0}[\bar{S}]$ is isomorphic to the contracted semigroup algebra $R_{0}\left[S^{\otimes}\right]$. For $J \in$ $S / 2$, we denote $\bar{J}=\{\bar{x} \mid x \in J\} \cup\{0\}$. It is easy to check that $(\bar{J}, *)$ is a subsemigroup of $\bar{S}$ isomorphic to the semigroup $J^{\otimes}$. So, for any $J, K \in S / 2$, we have

$$
\bar{J} * \bar{K} \begin{cases}\subseteq \bar{J} & \text { if } K=J, \\ =0 & \text { otherwise. }\end{cases}
$$


For Theorem 1.1, it remains to prove the following lemma.

Lemma 2.3. $R[S] \cong R_{0}[\bar{S}]$.

Proof. We consider the mapping $\varphi: R[S] \rightarrow R_{0}[\bar{S}]$ given on the basis by $\varphi(s)=\sum_{t \leq s} \bar{t}(s \in S)$. Clearly, $\varphi$ is well defined. Of course, $\varphi$ and - may be regarded as the mappings of the ordered set $(S, \leq)$ into the additive group of $R_{0}[\bar{S}]$. Now, by applying the Möbius inversion theorem to the mappings $\varphi$ and $\bar{\bullet}$, we have

$$
\bar{s}=\sum_{t \leq s} \varphi(t) \mu(t, s)=\varphi\left(\sum_{t \leq s} t \mu(t, s)\right),
$$

where $\mu$ is the Möbius function for $(S, \leq)$. Hence $\varphi$ is surjective.

We will prove that $\varphi$ is injective. For $\alpha_{0}=\sum_{x \in S} p_{x}^{0} x \in R[S]$, we denote by $\operatorname{supp}\left(\alpha_{0}\right)$ the set $\left\{x \in S \mid p_{x}^{0} \neq 0\right\}$ and by $M\left(\alpha_{0}\right)$ the set of maximal elements in the set $\operatorname{supp}\left(\alpha_{0}\right)$ with respect to the partial order $\leq$. In recurrence, we define $\alpha_{n}=\alpha_{n-1}-\sum_{x \in M\left(\alpha_{n-1}\right)} p_{x}^{n-1} x$, where $\alpha_{n}=\sum_{x \in \operatorname{supp}\left(\alpha_{n}\right)} p_{x}^{n} x$. Let $\beta_{n}=\sum_{x \in \operatorname{supp}\left(\beta_{n}\right)} q_{x}^{n} x$ with $n=0,1,2, \ldots$ If $\varphi\left(\alpha_{n}\right)=\varphi\left(\beta_{n}\right)$, then by the definition of $\varphi, \sum_{x \in M\left(\alpha_{n}\right)} p_{x} \bar{x}+\Gamma_{\alpha_{n}}=\varphi\left(\alpha_{n}\right)=\varphi\left(\beta_{n}\right)=\sum_{y \in M\left(\beta_{n}\right)} q_{y}^{n} \bar{y}+\Gamma_{\beta_{n}}$, where $\Gamma_{\alpha_{n}}=\sum_{x \in M\left(\alpha_{n}\right)} \sum_{y \in S, y<x} p_{y}^{n} \bar{y}$ and $\Gamma_{\beta_{n}}=\sum_{x \in M\left(\beta_{n}\right)} \sum_{y \in S, y<x} q_{y}^{n} \bar{y}$, and hence $\sum_{x \in M\left(\alpha_{n}\right)} p_{x}^{n} \bar{x}=$ $\sum_{x \in M\left(\beta_{n}\right)} q_{x}^{n} \bar{x}$, thus $M\left(\alpha_{n}\right)=M\left(\beta_{n}\right)$ and $p_{x}^{n}=q_{x}^{n}$ for any $x \in M\left(\alpha_{n}\right)$. This can imply the following.

Fact 2.4. If $\varphi\left(\alpha_{n}\right)=\varphi\left(\beta_{n}\right)$, then $M\left(\alpha_{n}\right)=M\left(\beta_{n}\right)$ and by the definition of $\varphi, \varphi\left(\alpha_{n+1}\right)=\varphi\left(\beta_{n+1}\right)$.

By the definition of $\varphi$, the following facts are immediate.

Fact 2.5. $\alpha_{n}=\beta_{n}$ if and only if $M\left(\alpha_{n}\right)=M\left(\beta_{n}\right)$ and $\alpha_{n+1}=\beta_{n+1}$.

Fact 2.6. If $\varphi\left(\alpha_{n}\right)=\varphi\left(\beta_{n}\right)$ and $M\left(\alpha_{n}\right)=\operatorname{supp}\left(\alpha_{n}\right), M\left(\beta_{n}\right)=\operatorname{supp}\left(\beta_{n}\right)$, then $\alpha_{n}=\beta_{n}$.

Note that $\left|\operatorname{supp}\left(\alpha_{0}\right)\right|<\infty$ and $\operatorname{supp}\left(\alpha_{n+1}\right) \subseteq \operatorname{supp}\left(\alpha_{n}\right)$. We thus have a smallest integer $k$ such that $M\left(\alpha_{k}\right)=\operatorname{supp}\left(\alpha_{k}\right)$. Clearly, $\alpha_{k+1}=0$. This means that $k$ is the smallest integer $t$ such that $\alpha_{t+1}=0$. Similarly, there exists the smallest integer $l$ such that $\beta_{l+1}=0$ and $M\left(\beta_{l}\right)=$ $\operatorname{supp}\left(\beta_{l}\right)$. Now, assume $\varphi\left(\alpha_{0}\right)=\varphi\left(\beta_{0}\right)$. By using Fact 2.4 repeatedly,

$$
\varphi\left(\alpha_{1}\right)=\varphi\left(\beta_{1}\right), \quad \varphi\left(\alpha_{2}\right)=\varphi\left(\beta_{2}\right), \ldots, \quad \varphi\left(\alpha_{k+1}\right)=\varphi\left(\beta_{k+1}\right) .
$$

But $\varphi\left(\alpha_{k+1}\right)=0$, we have $\varphi\left(\beta_{k+1}\right)=0$ and by the definition of $\varphi, \beta_{k+1}=0$. Thus $k+1 \geq l+1$ by the minimality of $l$, and $k \geq l$. Similarly, $l \geq k$. Therefore $k=l$. Since $\varphi\left(\alpha_{k}\right)=\varphi\left(\beta_{k}\right)$, by Fact 2.6, we have $\alpha_{k}=\beta_{k}$ since $M\left(\alpha_{k}\right)=\operatorname{supp}\left(\alpha_{k}\right)$ and $M\left(\beta_{l}\right)=\operatorname{supp}\left(\beta_{l}\right)$. Again by the hypothesis $\varphi\left(\alpha_{0}\right)=\varphi\left(\beta_{0}\right)$, and by Fact 2.4, $M\left(\alpha_{0}\right)=M\left(\beta_{0}\right)$; and by (2.6), $M\left(\alpha_{1}\right)=M\left(\beta_{1}\right), M\left(\alpha_{2}\right)=$ $M\left(\beta_{2}\right), \ldots, M\left(\alpha_{k}\right)=M\left(\beta_{k}\right)$. By Fact 2.5, $M\left(\alpha_{k-1}\right)=M\left(\beta_{k-1}\right)$; and $\alpha_{k}=\beta_{k}$ imply $\alpha_{k-1}=\beta_{k-1}$; moreover, by using Fact 2.5 repeatedly, $\alpha_{k-2}=\beta_{k-2}, \ldots, \alpha_{1}=\beta_{1}$ and $\alpha_{0}=\beta_{0}$. We have now proved that $\varphi$ is injective.

Finally, for any $s, t \in S$, by (2.4), we have

$$
\bar{s} * \bar{t}= \begin{cases}\overline{s t} & \text { if } s, t \in J_{s t}, \\ 0 & \text { otherwise, }\end{cases}
$$


and by Lemma 2.2,

$$
\begin{aligned}
\varphi(s) * \varphi(t) & =\left(\sum_{x \leq s} \bar{x}\right) *\left(\sum_{y \leq t} \bar{y}\right) \\
& =\sum_{x \in J_{s t}, x \leq s} \sum_{y \in J_{s t}, y \leq t} \bar{x} * \bar{y} \\
& =\sum_{x \in J_{s t}, x \leq s} \sum_{y \in J_{s t}, y \leq t} \overline{x y} .
\end{aligned}
$$

Moreover, by Lemma 2.2, we have

$$
\begin{aligned}
\varphi(s t) & =\sum_{u \leq s t} \bar{u}=\sum_{x \in J_{s t}, x \leq s} \sum_{y \in J_{s t}, y \leq t} \overline{x y} \\
& =\sum_{x \leq s, x \in J_{s t}} \sum_{y \leq t, y \in J_{s t}} \bar{x} * \bar{y}=\varphi(s) * \varphi(t) .
\end{aligned}
$$

Thus $\varphi$ is a homomorphism of $R[S]$ into $R_{0}[\bar{S}]$. Consequently, $\varphi$ is an isomorphism of $R[S]$ onto $R_{0}[\bar{S}]$.

\section{Acknowledgment}

The research is supported by the NSF of Jiangxi Province, the SF of Education Department of Jiangxi Province, and the SF of Jiangxi Normal University.

\section{References}

[1] A. H. Clifford and G. B. Preston, The Algebraic Theory of Semigroups, vol. 1 of Mathematical Surveys, no. 7, American Mathematical Society, Providence, RI, USA, 1961.

[2] B. Steinberg, "Möbius functions and semigroup representation theory," Journal of Combinatorial Theory, vol. 113, no. 5, pp. 866-881, 2006.

[3] K. S. S. Nambooripad, "The natural partial order on a regular semigroup," Proceedings of the Edinburgh Mathematical Society, vol. 23, no. 3, pp. 249-260, 1980.

[4] K. S. S. Nambooripad, "Structure of regular semigroups. I," Memoirs of the American Mathematical Society, vol. 22, no. 224, p. vii+119, 1979. 


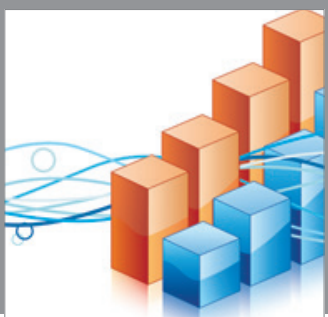

Advances in

Operations Research

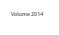

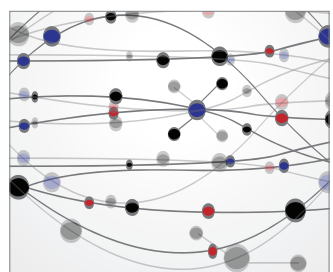

\section{The Scientific} World Journal
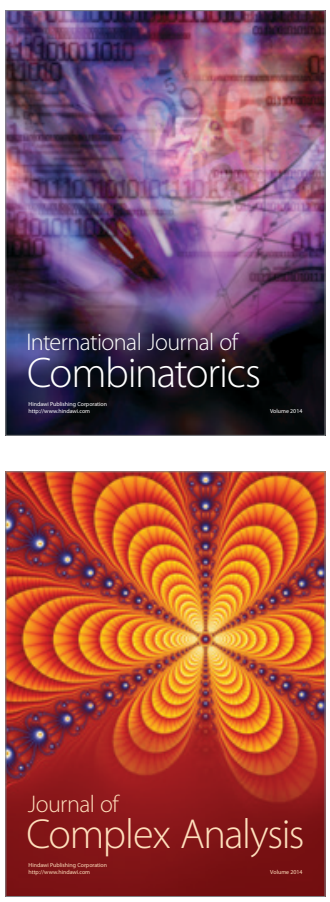

International Journal of

Mathematics and

Mathematical

Sciences
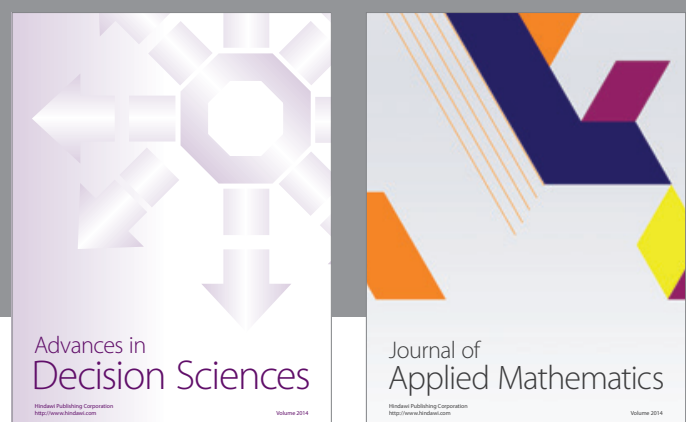

Journal of

Applied Mathematics
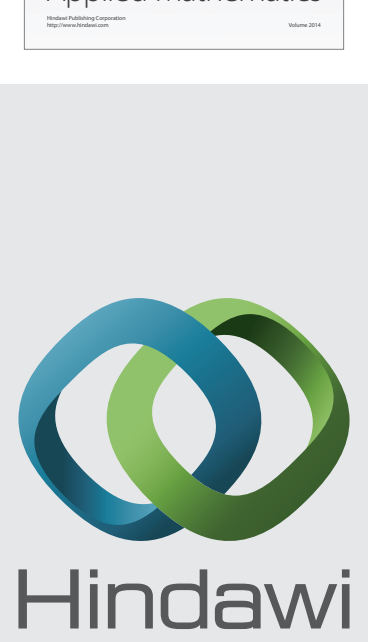

Submit your manuscripts at http://www.hindawi.com
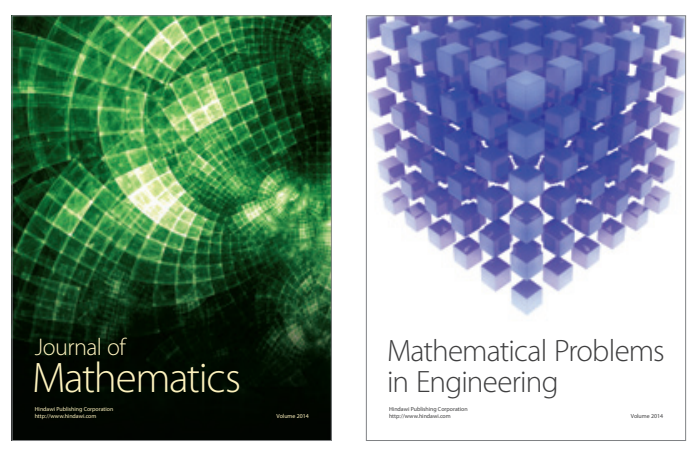

Mathematical Problems in Engineering
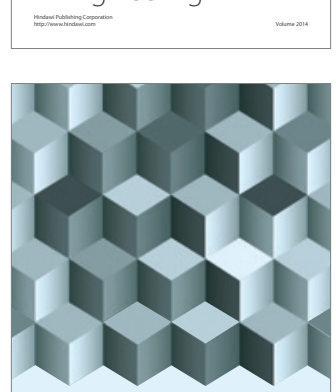

Journal of

Function Spaces
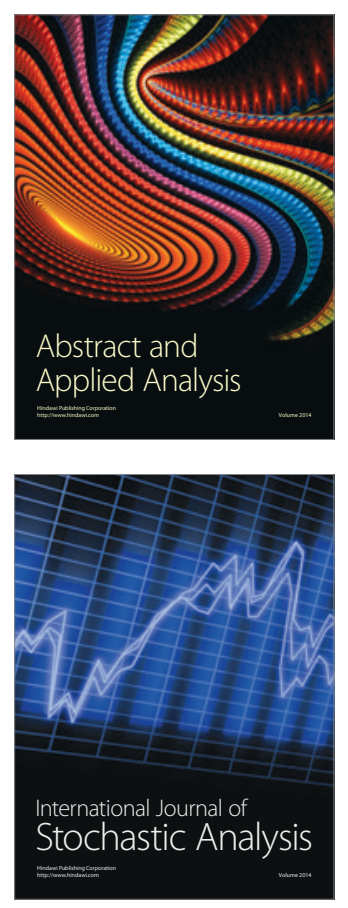

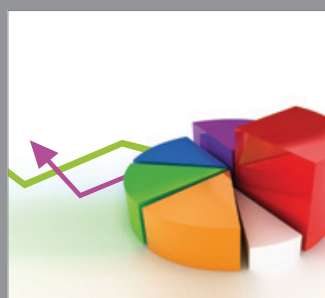

ournal of

Probability and Statistics

Promensencen
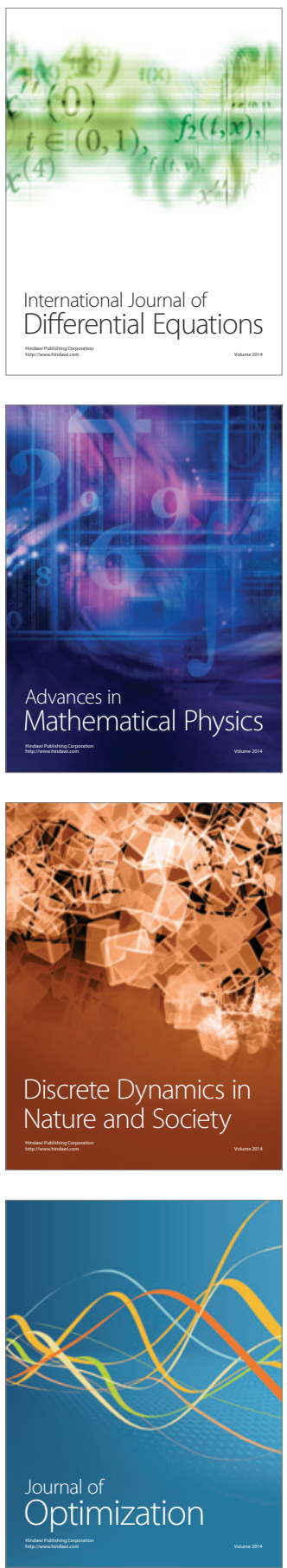